Oncology 1993;50(suppl 2):I-III

\title{
Contents, Supplement 2, 1993
}

Pico, J.L.; Ibrahim, A.; Castagna, L.; Bourhis, J.H.; Chazard, M.; Maraninchi, D.; Droz, J.P. Escalating High-Dose Carboplatin and Autologous Bone Marrow Transplantation in Solid Tumors

47

Author and Subject Index

53 